\title{
Nutrient content of products purchased following the implementation of the "Healthier Vending Policy" in Ireland.
}

\author{
Olivia Porter ${ }^{1}$, Isobel Stanley ${ }^{1}$, Agatha Lawless ${ }^{2}$, Sarah O’Brien ${ }^{2}$, Cecily C. Kelleher ${ }^{1}$, \\ Celine M. Murrin ${ }^{1}$ and Silvia Bel-Serrat ${ }^{1}$ \\ ${ }^{1}$ National Nutrition Surveillance Centre, School of Public Health, Physiotherapy and Sports Science, University \\ College Dublin, Dublin, Ireland and \\ ${ }^{2}$ Healthy Eating \& Active Living Programme, Health Service Executive, Dublin, Ireland
}

\begin{abstract}
Introduction: The food environment influences food choices made by populations. On-the-go snacking has become an integral part of modern Irish life and must be considered when shaping such environments, considering that the Republic of Ireland population consume an average of 2.5 snacks daily, with $22 \%$ of snacking occasions occurring outside the home. This has driven the increased prevalence of snack food vending machines which typically stock unhealthy products that are high in energy, fat and sugar. The Health Service Executive (HSE) implemented the Healthier Vending Policy in 2015 to increase the availability of healthier products across HSE premises. This research examined the nutrient content of snacks and beverages purchased in vending machines on HSE premises following the implementation of the HSE Healthier Vending Policy.
\end{abstract}

Materials and Methods: Vending machines product and sales data were obtained from two vending machines companies at baseline in 2015 and following policy implementation in 2016. Product labels and online product information were used to obtain the energy and nutrient contents. The products were assigned to a snack or beverage category; nuts, mints, cereal bars, chocolates, sweets, crisps, waters, juices, carbonated drinks and other. New variables were computed, descriptive analyses were undertaken and Wilcoxon-signed rank tests performed using SPSS Statistics 24. Statistical significance was set at $\mathrm{p}<0.05$.

Results: Snacks and beverages sold in 295 vending machines across 89 HSE premises were analysed. There were statistically significant decreases in median energy sold per packet $(9.7 \%)$ and per $100 \mathrm{~g}(7.1 \%)$ following policy implementation. Reductions in total fat $(53.2 \%)$, saturated fat $(32.4 \%)$, sugar $(10.0 \%)$ and sodium $(20.0 \%)$ sold also occurred. When snacks were analysed separately the findings were less positive, as a statistically significant increase in energy sold per packet and an increase in total fat sold per $100 \mathrm{~g}$ occurred. The findings in relation to beverages were mixed. The effect of the policy varied across snack categories, with particularly large reductions in energy sold per packet in carbonated drinks $(54.0 \%)$ and cereal bars $(44.2 \%)$.

Discussion: Adecrease in the amount of energy, total fat, saturated fat, sugar and sodium sold was observed following the implementation of the policy. The HSE Healthier Vending Policy seemed to promote a healthier environment. It is recommended that this policy continues to be implemented and is reviewed to ensure the best possible outcomes nationally. It is also advisable to bring this policy to other settings to combat obesogenic environments and promote public health.

\section{Conflict of Interest}

There is no conflict of interest. 